\title{
Aspectos geoéticos en la docencia del ordenamiento y la gestión del territorio
}

\author{
Juan Gregorio Rejas Ayuga, Joaquín Bosque Sen- \\ dra,José Antonio Malpica† ,Francisco Maza Vázquez; \\ Adolfo Dalda Mourón, María Luisa Soriano Sanz, María \\ Guadalupe Rodríguez Díaz, Juan Luís Bermúdez, \\ Fernando Cerezal, Roberto Goycoolea Prado, Francisco \\ Javier González Matesanz, María Concepción Alonso \\ Rodríguez, Francisco Javier Gómez Martínez, Jesús \\ Martínez-Frías
}

\section{Resumen:}

La Geoética es la disciplina que interpela y trata desde un enfoque ético las materias profesionales y de investigación involucradas en las Ciencias de la Tierra y Planetarias en su conjunto. Desde su irrupción a finales del pasado siglo hasta la actualidad, ha experimentado una importante notoriedad reconocida por la AGID (Association of Geoscientists for International Development), IUGS (International Union of Geological Sciences) y otras organizaciones internacionales en consonancia con los avances tecnológicos, de la información geográfica y geocientíficos en general cada vez más interrelacionados con el mundo abiótico.

En el presente artículo se analizan problemáticas y retos geoéticos en un contexto docente del ordenamiento y gestión del territorio. Se exponen diversos aspectos relacionados con códigos de conducta y buen hacer que han aflorado o ha tenido que abordar el profesorado de la Maestría desarrollada conjuntamente por la Universidad Nacional Autónoma de Honduras (UNAH) y la Universidad de Alcalá (UAH) en sus sucesivas ediciones. Finalmente se realiza una aproximación desde las distintas áreas de conocimiento al tratamiento conceptual, profesional y de investigación de la Geoética en un ámbito centroamericano, cuyo resultado último implique aportar respuestas ante nuevos retos globales.

Palabras clave: Geoética/Ciencias de la Tierra/Tecnología/Información/Docencia. 


\section{Abstract:}

The Geoethic is the discipline that comes from challenges and ethical approach subjects and professionals research involved in the Earth Sciences and Planetary together. Since its emergence in the late last century to the present, has undergone a major notoriety recognized by AGID (Association of Geoscientists for International Development), IUGS (International Union of Geological Sciences) and other international organizations in line with technological advances and geographic information increasingly interrelated with the abiotic world.

This article discusses problems and challenges geoethic in a teaching context for organization and management of the territory. It presents various aspects of codes of conduct and good work that have emerged or have had to address the faculty of the Master developed jointly by the National Autonomous University of Honduras (UNAH) and the University of Alcala (UAH) in successive editions. Finally, an approximation from the different areas of knowledge conceptual treatment, professional and research in a field geoethic Central, with the end result involves providing answers to new global challenges.

Keywords: Geoethic/Earth Science/Technology/Information/Teaching.

Juan Gregorio Rejas Ayuga ${ }^{1,5},($ rejasaj@inta.es), (juangregorio.rejas@upm.es); Joaquín Bosque Sendra 22, (joaquin.bosque@uah.es); José Antonio Malpica† 2; Francisco Maza Vázquez², (francisco.maza@uah.es); Adolfo Dalda Mourón², (adolfo.daldamouron@gmail.com); María Luisa Soriano Sanz², (maria.soriano@sepes.es); María Guadalupe Rodríguez Díaz ${ }^{2}$, (guadalupe.rodriguez@uah.es);Juan Luís Bermúdez², (jlbermudez@beritec.es); Fernando Cerezal2, (nicoop.academico@uah.es); Roberto Goycoolea Prado2, (roberto.goycoolea@ uah.es); Francisco Javier González Matesanz ${ }^{2,4}$ (fjgmatesanz@fomento.es); María Concepción Alonso Rodríguez z', (mconcepción.alonso@uah.es); Francisco Javier Gómez Martínez ${ }^{6}$, (igitur40@msn.com); Jesús Martínez-Frías ${ }^{3,5}$, (jmfrias@cab.inta-csic.es). ${ }^{1}$ Instituto Nacional de Técnica Aeroespacial, INTA. ${ }^{2}$ Universidad de Alcalá de Henares, UAH. ${ }^{3}$ Centro de Astrobiología, CSIC-INTA. ${ }^{4}$ Instituto Geográfico Nacional, IGN. ${ }^{5}$ Universidad Politécnica de Madrid, UPM. ${ }^{6}$ Universidad Complutense de Madrid. †Dedicatoria: un recuerdo especial para nuestro admirado compañero y amigo, profesor José Antonio Malpica, fallecido el 1 de diciembre de 2012. Su ejemplo siempre quedará en nuestra memoria. 


\section{INTRODUCCIÓN}

El camino por el que se llega a la ética (Simondon, 1989; González, 2000) en sus diferentes variantes es muchas veces consecuencia de una pregunta o preguntas iniciales que se le plantean al individuo en el ejercicio de la acción, sea ésta su profesión, su formación o su ámbito personal. Es por lo tanto la búsqueda de respuestas ante el devenir, ante situaciones concretas, novedosas o cambiantes, lo que obliga al ser humano, podríamos decir que de una manera innata, a entrar en disposición intelectual para saciar esa necesidad que irrumpe. La Geoética no es un caso diferente en lo que se refiere al impacto que producen los nuevos fenómenos en el posicionamiento ético y moral (Nemec, 2012).

Existe un esquema fundamental que opera en este sentido y que relaciona el bloque problema/pensamiento cognitivo/praxis en un flujo secuencial que puede o no cerrarse. La Antropología, la Sociología y otras materias afines (Cliffort, 1995; Herbert, 1992; López, 2007; Swatz et al., 1994) apuntalan desde hace décadas que precisamente esta capacidad de discurrir y desarrollar soluciones abstractas es una de las claves del éxito de adaptación y supervivencia del ser humano como especie.

Es importante recalcar ese parámetro, esa causa inicial, es decir, el binomio cambio-adaptación. El conocimiento, las sociedades, el individuo no permanecen fijos. En tiempos como los actuales, en los que se experimenta un vertiginoso avance tecnológico, son mucho más apreciables estos efectos en la sociedad y en su relación con el entorno. Los espacios naturales bióticos y abióticos no permanecen inalterables. ¿Cuál es la tolerancia establecida y admitida por el ser humano a estos cambios? Una respuesta inmediata, situando en el centro del universo al ser humano y anteponiendo nuestra propia supervivencia como especie, podría ser que no existen límites. Pero esta premisa se demuestra insuficiente. La hipótesis de Gaia (Lovelock, 2003) como teoría controvertida que considera a nuestro planeta un ser vivo en el cual todo interacciona, supone una posible respuesta científica a los continuos desajustes naturales y humanos que se vienen observando en los últimos tiempos, y que en un principio parecieran responder a causas atomizadas. ¿Cuál es entonces el mecanismo por el que vamos buscando y encontrando respuestas a medida que irrumpen nuevos retos?

A lo largo del siglo pasado y en lo que llevamos de este, las Ciencias de la Tierra y Planetarias, también llamadas Geociencias, han experimentado un desa- 
rrollo espectacular, unido indisolublemente al salto cualitativo que ha experimentado en este mismo período la Tecnología, en lo que se asume ya como un nueva revolución de la humanidad. Inevitablemente se tienen que producir nuevas relaciones entre estas ciencias y los espacios, físicos o abstractos, estudiados. También surgen inquietudes y roces, preguntas sin respuesta, que desde una perspectiva responsable como científicos es necesario abordar. La Geoética pretende en este sentido analizar desde un enfoque ético los aspectos y conflictos que afloran en la interacción de las Geociencias y su puesta en práctica en distintas áreas docentes, profesionales y de investigación, representando ciertamente, una oportunidad para los científicos en la toma de conciencia de su papel y responsabilidad sociales.

En el presente artículo se realiza una aproximación a la Geoética, analizando por separado para luego relacionar su implicación en los diferentes ámbitos. Se exponen los contactos con la ética y las experiencias de un grupo multidisciplinar de docentes y profesionales en el contexto de las Tecnologías de la Información Geográfica, la Planetología, el Ordenamiento del Territorio y la Cooperación para el Desarrollo. Se establecen finalmente conclusiones encaminadas a observar los retos futuros y contribuir a construir acuerdos para un marco ético en las Geociencias.

\section{ANTECEDENTES ACTUALES. LA BIOÉTICA COMO EJEMPLO.}

El término Geoética comienza a aparecer en los foros científicos a finales del siglo XX. Inicialmente promovida desde la Geología, nace en 1991, considerándose al Dr. Vàclav Nemec (Martínez-Frías, 2008) como el padre de esta nueva disciplina. No es hasta 2004 cuando se asienta su institucionalización en forma de uno de los grupos de trabajo impulsado por la Asociación de Geocientíficos para el Desarrollo Internacional (AGID).

Nemec y Nemcoca formulan la Geoética inicialmente como una nueva disciplina en el marco de las Ciencias de la Tierra, que vincula ética y geología, y engloba aspectos científicos y sociales desde enfoques prácticos y teóricos (Nemec, 1992; Nikitina, 2012). Ampliando e integrando esta formulación primera, Martínez-Frías (Martínez-Frías, 2008) propone una definición formal de la Geoética como una disciplina clave en el campo de las Ciencias de la Tierra y de las Ciencias Planetarias, que involucra diferentes aspectos de carácter científico, tecnológico, metodológico y sociocultural (por ejemplo, sostenibilidad, desarrollo, museología), pero también la necesidad de considerar protocolos apropiados, problemas de integridad científica 
y códigos de buenas prácticas respecto al estudio del mundo abiótico. Los estudios sobre geología planetaria y astrobiología también requieren un enfoque geoético.

Un caso antecesor, en gran medida extrapolable al mundo abiótico, lo supone la amplia actividad que desde la Bioética se viene desarrollando internacionalmente desde la década de los 70 del siglo pasado (Potter, 1970). Si bien son más evidentes y directas las repercusiones para el respeto a la vida en este caso, este hermano mayor ha sentado las bases para establecer marcos adecuados desde la ética en un ámbito científico y profesional.

La satisfactoria e ingente experiencia de la Bioética en la docencia (Couceiro-Vidal, 2008; Mellado, 2004; Escobar et al., 2008), la investigación, la sociedad y la política (Keyuex et al., 2004) puede ser un buen ejemplo a seguir para la Geoética, o cuando menos, un precursor del que aprender de sus aciertos y errores.

\section{GEOÉTICAY PROFESIÓN.}

Es el ejercicio de la profesión de las Geociencias el marco en el que originalmente tienen lugar problemáticas éticas. La explotación de recursos minerales, su protección y moderación de consumo de fuentes no renovables de la Tierra, da origen de facto a la Geoética en el ámbito profesional (Nemec, 2012). Cuando el individuo entra en relaciones profesionales, las dimensiones de la Geoética deben elevarse a los requerimientos de la mentalidad profesional, porque ésta propicia profesiones con alto nivel de conciencia moral. Geoética y mentalidad profesional, son sólo algunos aprendizajes insustituibles. La mentalidad profesional exige una actitud más proactiva para mejorar el campo de la profesión ateniéndose a la Geoética (González y Martínez-Frías, 2011). ¿Cómo influye o afecta la ética en el ámbito y ejercicio profesional?

A las causas iniciales se han unido otras prioridades, consecuencia de los resultados obtenidos en la puesta en práctica de temas relacionados con los desastres naturales y el impacto demoledor que su creciente intensidad provocan. Casos recientes como el terremoto de L'Aquila (Italia) en 2009, el terremoto de Lorca (España), el maremoto y posterior tsunami de Fukushima (Japón) o el devastador tsunami de Indonesia de 2004, son claros ejemplos tanto por sí mismos como por las consecuencias que producen, de la necesidad de valoraciones éticas que los profesionales de las Geociencias están abocados a plantearse. No habría que 
olvidar el caso del terremoto de Chile en 2010, que evidenció escasez de estudios geotécnicos, incluso en torres de vivienda, lo que unido a una falta de vinculación entre investigadores universitarios y legisladores, vio agravados los efectos propios de la catástrofe natural.

La modelización mediante sistemas de información geográfica, generación de alertas tempranas ante catástrofes y normas sismoresistentes (u otras) para la construcción, ha supuesto un importantísimo avance, que ha repercutido positivamente en la eficiencia de las medidas a adoptar ante estas situaciones. Bien, pero a la par que se ha producido esta mejora y ante casos nuevos, surgen aspectos éticos a considerar relacionados con protocolos de actuación, tiempos de ejecución y responsabilidades profesionales. El caso de L'Aquila mencionado, por reciente y cercano, es quizás paradigmático de esta situación. Si bien los estudios profesionales pueden preveer un riesgo inminente de catástrofe, es necesario coordinar estos resultados técnicos con decisiones políticas sujetas a protocolos de actuación consensuados en base a la experiencia previa.

El ámbito profesional del Patrimonio es otro elemento sujeto a grandes tensiones y riesgos. Un enfoque ético en las geociencias que trabajan en patrimonio debe ser así mismo considerado. ¿Los espacios geográficos y geológicos tienen que ser protegidos?¿Qué soluciones éticas podemos aportar si se plantea un proyecto, por ej. la cuestionada construcción de la presa en la Patagonia chilena, que afectará irremediablemente a estos lugares?
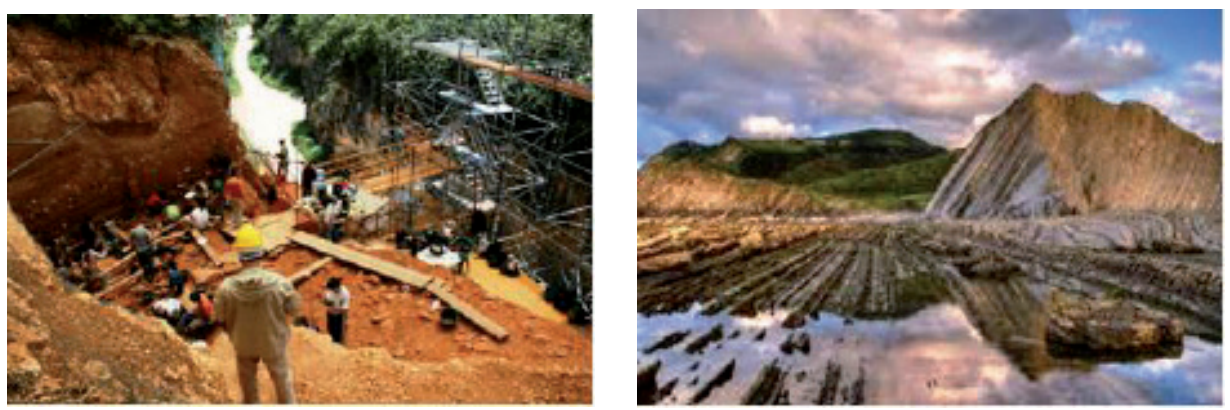

FIGURA 1. Yacimiento de Atapuerca en Burgos, España, declarado Patrimonio de la Humanidad (Izqda.) y sistema de Flysch en el Geoparque de la Costa Vasca (dcha.) 
El concepto de Patrimonio es amplio, abarcando lo material y lo inmaterial. Cabe mencionar la reciente declaración de UNESCO como Patrimonio Cultural de la Humanidad también del Patrimonio Geológico, en el que se incluyen espacios geológicos únicos, meteoritos, rutas geológicas, etc. (Figura 1).

Existe la creencia generalizada de que la vida animal y vegetal resulta siempre más vulnerable a los cambios o las agresiones que la geología y los espacios geográficos. Las montañas y las rocas parecen indestructibles y creemos que seguirán inalterables durante miles de años. Sin embargo, la naturaleza biológica es flexible y casi siempre tiene una cierta capacidad para adaptarse a los cambios, mientras que muchos elementos abióticos no pueden acomodarse a cambios 0 agresiones. Es por lo tanto una necesidad urgente transmitir conciencia del valor de la geología y los espacios geográficos para conservar todos sus elementos. Es necesario insistir sobre las consecuencias irreversibles que tendría la destrucción de la mayoría de los enclaves con valor espacial.

A esta se añade la acuciante problemática del expolio del patrimonio natural y cultural, geológico incluido (meteoritos, minerales, fósiles, etc.). Tarea que tiene que ser abordada también desde un enfoque práctico por la Geoética (Martínez-Frías y Verde, 2010), desarrollando protocolos para su protección y tecnologías de las Ciencias de la Tierra que permitan su registro, inventariado y monitorización como acciones iniciales.

Desde hace varias décadas se ha asentado otro aspecto a considerar en relación con el auge en la exploración de otros planetas. Organismos científicos tan relevantes como las agencias espaciales estadounidense NASA (National Aeronautics and Space Administration) y europea ESA (European Space Agency) o el Centro de Astrobiología ( $C A B$ ) del Instituto Nacional de Técnica Aeroespacial y el Consejo Superior de Investigaciones Científicas de España, se vienen planteando los aspectos relativos a la alteración físico-química y las metodologías no invasivas para la investigación de determinados espacios planetarios (Martínez-Frías et al., 2012). Podríamos aventurar que se ha llegado a un punto de encuentro, con diferentes matices y controversias, sobre la necesidad de establecer protocolos y procedimientos consensuados para intentar no repetir fuera de la Tierra los errores cometidos en el pasado en nuestro propio planeta. Por un lado se pretende analizar las implicaciones éticas de la investigación humana que tiene, quizás sin camino de vuelta, para los planetas o cuerpos celestes explorados. Por otro lado, se plantea 
hasta qué punto afecta la interacción humana en los propios resultados de la investigación planetaria, en lo que puede considerarse como analogía macro física con el conocido principio de Incertidumbre de Heisemberg. ¿Hasta qué punto el propio estudio de un elemento no altera el elemento estudiado?

Otro aspecto que hay que introducir en el debate es el de la seguridad (Figura 2) en el ejercicio de las profesiones de las geociencias. Seguridad de quien ejerce y seguridad de quien puede verse afectado por el ejercicio de las geociencias. En el ámbito de geodesia, tiene relación el hecho de que exista una "Ley de señales geodésicas y geofísicas", sin embargo, por su antigüedad, no contempla las relaciones con el medio ambiente y entre diversos organismos implicados en las responsabilidades éticas de la medida, investigación y gestión del medio. Si bien se trata de una legislación acorde con las necesidades de su tiempo, hay aspectos que no se han cumplido con el rigor previsto, aunque sí está resultando útil para atender a las modificaciones demandadas por particulares y otros organismos.

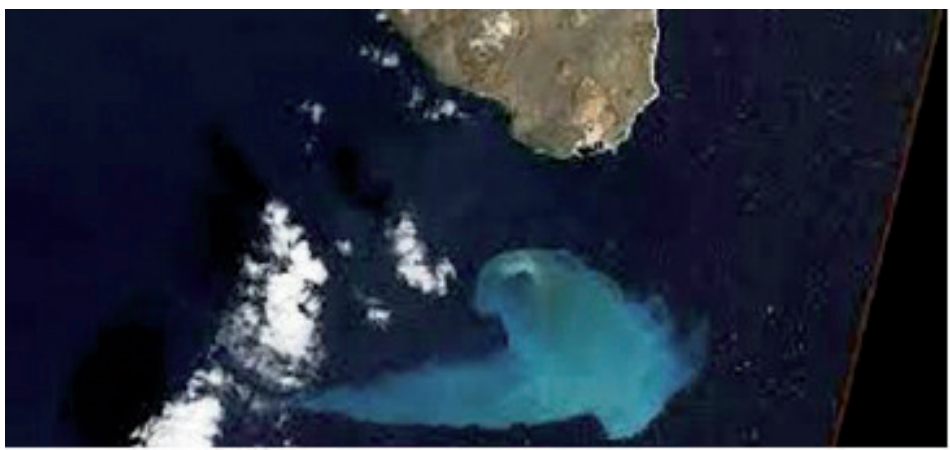

FIGURA 2. Erupción oceánica de 2012 en la isla del Hierro, España. Mejor imagen del año para NASA.

Si bien, el problema es complejo y transversal, un punto de partida interesante para una ética normativa o deóntica lo puede suponer los tres principios normativos (Méndez y Gómez, 2008) de Responsabilidad, Precaución y Autocontención que, con una formulación diferente, usaba Hans Jonas. Resulta evidente que, al menos entendido desde un enfoque ético, no se aplicarían específicamente a los individuos, sino también a las instituciones o a los gobiernos, algo fundamental para una Geoética que además, implicaría una responsabilidad intergeneracional. 


\section{GEOÉTICA Y DOCENCIA.}

La ética es necesaria en la formación de los investigadores, insustituible para el pensamiento crítico, imprescindible como la lógica para indagar sobre fenómenos trascendentes para la Humanidad (Gardner, 2005). La Geoética en un enfoque docente, debe relacionarse con el concepto de Antropoética, introducido por el filósofo Edgar Morin (Morin, 1999) creador del paradigma del "pensamiento complejo". Se concibe de esta manera al hombre como "hombre ético", es decir un hombre con obligaciones morales para con él, sus semejantes y la naturaleza. Se analizan los saberes fundamentales para una educación bajo esta premisa, que en un sentido amplio viene a significar una actitud y una forma de vida en donde el objetivo principal es humanizar a la sociedad y al hombre.

La experiencia en el desarrollo docente de las Geociencias en las materias mencionadas, aconseja la necesidad de contemplar aspectos Geoéticos en los programas formativos. Cada vez de manera más asidua se producen planteamientos éticos en los másteres y carreras de grado en Geociencias. Si bien desde algunas instituciones como AGID (Association of Geoscientists for International Development) e IAEGTH (International Association for Geoethics, http://tierra.rediris.es/IAGETH/) se debate ya algunos elementos incipientes que permiten abrigar bue-nas esperanzas para un planteamiento riguroso en materias docentes, no se puede afirmar que en estos momentos exista un marco adecuado ni el bagaje necesario para definir mínimamente la docencia en Geoética de tal manera que se pueda materializar en asignaturas incorporadas a los programas formativos en la universidad. Queda todavía camino por recorrer para alcanzar ese estadio inicial. Esto no es óbice para ir poniendo encima de la mesa cuestiones tales como: ¿Cuáles son los comienzos que tiene que tener la docencia en Geoética? ¿Cuál es el ámbito del que se debe ocupar la Geoética en la docencia? ¿Qué carácter tiene que tener, debe ser obligatorio u optativo? ¿Qué repercusión puede tener en los universitarios? En lo referente a estos aspectos docentes de la Geoética, es necesario añadir la necesidad de plantear la inclusión en las enseñanzas de las relaciones entre participación de la población en la Ordenación del Territorio y los distintos enfoques de justicia, espacial y ambiental, en la localización de las actividades humanas (Bosque et al., 2001).

Mención especial tiene la relación entre docencia, ética y matemática que los clásicos como Aristóteles nos transmitieron, dejando unas primeras y más que 
interesantes reflexiones sobre la técnica en su Ética a Nicómaco: el inicio del conocimiento es la sorpresa o admiración (thauma) ante la conciencia de un problema. Esta tradición de enseñanza entre ética y lenguaje matemático debe ser recogida en las Geociencias y materializada también por la Geoética. Así se ha venido manifestando, si bien no de manera consciente ni formal, sí en la práctica, como demuestra el bagaje de aprendizaje transmitido por ilustres matemáticos (Riechmann, 2003), entre los que tiene un lugar especial José Antonio Malpica por su imborrable experiencia docente e investigadora, reflejada en una dilatada obra de trabajos científicos, muchos de los cuales relacionan implícita o explícitamente ética y Ciencias de la Tierra. Entre ellos, destacamos la aportación en modelos matemáticos avanzados para una mejor formulación de mapas de riesgo natural (Ahamdanech et al., 2003).

\section{CENTROAMÉRICA, UNA OPORTUNIDAD TAMBIÉN PARA LA GEOÉTICA.}

Los autores del artículo representan un grupo multidisciplinar que ha mantenido y mantiene una profunda y estrecha relación profesional, docente y afectiva con Centroamérica. Una dilatada y rica experiencia de Cooperación con Centroamérica en investigación y docencia, de resultados tan esperanzadores, asenta la bases para plantear desde un inicio y de manera correcta una colaboración honesta en el espacio geoético.

La maestría de Ordenamiento y Gestión del Territorio que desde 2004, y a punto ya de abrirse su $4^{a}$ edición, viene desarrollándose conjuntamente entre la Universidad de Alcalá y la Universidad Nacional Autónoma de Honduras, ha supuesto un adecuado escenario en el que inconscientemente se han venido planteando aspectos geoéticos.

Las actividades de Ordenamiento y Gestión del Territorio forman parte de los otros diez proyectos de cooperación universitaria para el desarrollo que se vienen desarrollando por el Programa de Cooperación con Centroamérica de la Universidad de Alcalá (Figura 3). Esta cooperación, iniciada en 1988, se ha caracterizado por dos rasgos muy definidos: apoyar a las universidades centroamericanas en su fortalecimiento institucional como agentes de desarrollo de sus países a través de la actualización para la docencia y la investigación, y por promover la solidaridad de todos los sectores de la comunidad universitaria (profesores, técnicos y estudiantes) para apoyar los procesos de cambio en Centroamérica. Los proyectos, en consecuencia, han estado orientados al desarrollo de la autonomía de las universi- 
dades centroamericanas, a la concentración temática sostenida en el tiempo de programas y planes, al seguimiento y evaluación permanentes, a la coordinación intra e interuniversitaria con una misma filosofía de cooperación solidaria y a un enfoque aplicado a la resolución de problemas sociales en la región centromericana.

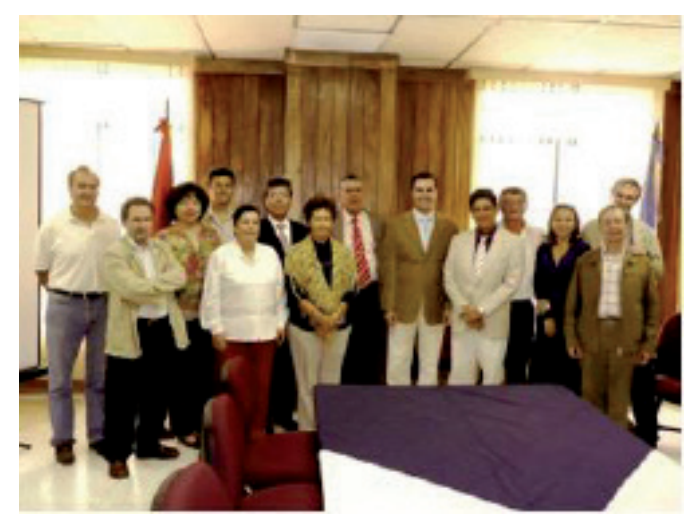

FIGURA 3. Reunión de la Red Interuniversitaria de Cooperación para el Desarrollo de Centroamérica -Red GIRA(http://redgira.unanleon.edu.ni/) en 2009.

Si bien las problemáticas geoéticas en su origen tienen un carácter principalmente ambiental, en la Ordenación del Territorio se relacionan además con las cuestiones humanas y de bienestar social, adquiriendo estos un peso mayor en lo referente a las repercusiones de la gestión de un territorio. Una cuestión general a plantear es la manera más adecuada y ética de realizar la Ordenación del Territorio: ¿es una tarea simplemente técnica?, un técnico/científico con herramientas de optimización (modelos de localización óptima, etc) puede definir dónde deben situarse las cosas en el territorio, o es más adecuado y más ético elaborar un sistema de participación ciudadana para definir la localización de las cosas y la formulación de planes de ordenación del territorio.

En no pocas ocasiones profesores y coordinadores en las materias del ordenamiento y gestión del territorio se han visto en la coyuntura de establecer prioridades en la investigación de espacios geográficos, sin el apoyo de una deontología convenientemente desarrollada. No se trata de un aspecto menor si se tiene en cuenta la repercusión que estas decisiones puedan producir en las áreas y comunidades, muchas de las cuales se encuentran en vías de desarrollo. Tenemos que preguntarnos si es más adecuado éticamente iniciar la investigación en lugares 
desfavorecidos o por el contrario fortalecer la investigación ya iniciada de espacios-lugares en los que ya se han invertido recursos (Figura 4). La Geoética debe involucrarse también en la respuesta no sencilla a estas preguntas. Para responder a estas cuestiones, genéricamente formuladas como disyuntiva entre justicia espacial y justicia ambiental, se puede adoptar la aproximación recogida en las numerosas publicaciones sobre SIG participativos (Bosque y Gómez, 2011; Bosque y Rivas, 2011; Riechmann, 2004).
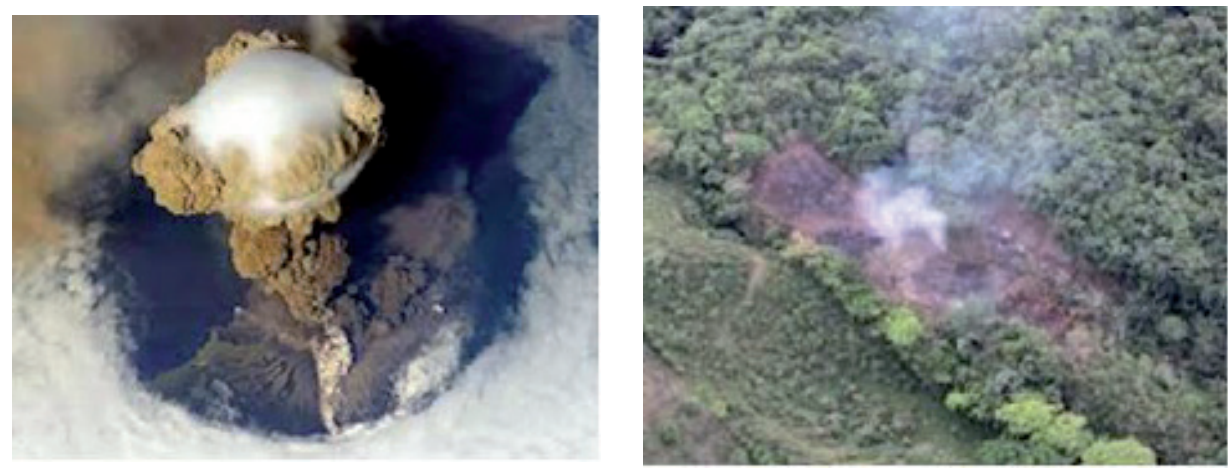

FIGURA 4. Erupción del volcán San Cristóbal, Nicaragua, (izda.) e impacto de meteorito en Honduras .(dcha.) ambos en 2012.

\section{CONCLUSIONES Y RETOS GLOBALES.}

En el presente artículo se ha realizado una introducción sobre la Geoética, cuya inicial pretensión ha sido dar a conocer a esta nueva disciplina nacida como vínculo entre la ética y las Ciencias de la Tierra y Planetarias. Retos actuales sobre deontología en la profesión, consensos de buenas prácticas, relación con la sociedad y la política, protocolos de actuación o implementación docente, quedan planteados. Se ha expuesto un enfoque teórico y otro práctico no contradictorios, sino al contrario, necesariamente convergentes.

Se han aproximado aspectos geoéticos incipientes en ámbitos profesionales y docentes, enfatizando las relaciones éticas de las Geociencias en un contexto del Ordenamiento y la Gestión del Territorio. En referencia a esta materia en particular, se matiza la especial disyuntiva sobre la justicia espacial y justicia ambiental que afecta al Ordenamiento y la Gestión del Territorio.

Se ha hecho una reflexión especial sobre Centroamérica, como espacio 
social, cultural y geográfico rico y dinámico, en base a los contactos con aspectos éticos experimentados por un grupo multidisciplinar de profesionales y docentes en esta región de la Tierra. Los autores concluyen que hay que establecer, y en su caso reforzar, los lazos interuniversitarios para iniciar un planteamiento docente sólido en materia de Geoética.

Finalmente, los autores consideran necesario contemplar y promover planteamientos geoéticos ante retos y problemáticas globales, de tal manera que el avance tecnológico beneficioso que experimentamos en las últimas décadas, suponga así mismo un fortalecimiento consistente y sostenible para la humanidad y las generaciones futuras.

\section{Referencias Bibliográficas.}

- Ahamdanech, I., Alonso, C., Bosque Sendra, J., Malpica, J.A., Martín-Loeches, M., Pérez, E y Temiño, J. (2003). "Un procedimiento para la elaboración de mapas de riesgos naturales aplicado a Honduras", Anales de Geografía de la Universidad complutense de Madrid, (2003), n²3, p. 55-73.

- Bosque Sendra, Joaquín y Gómez Delgado, Monserrat (2011). "Sistemas de Ayuda a la Decisión Espacial para la Ordenación del territorio." En: Ma Cristina Pineda de Carias y Bosque Sendra, J. (Edits.) Actas. I Congreso internacional de Ordenamiento territorial y tecnologías de la información geográfica. Servicio de publicaciones de la UAH, Alcalá de Henares, Madrid, 2011, 33 p. ISBN: 97884-8138-920-3.

- Bosque Sendra, Joaquín y Rivas González, Fredy (2011). "Propuesta Metodológica para el Estudio de la Justicia Ambiental Mediante el Uso de Herramientas SIG". En: Ma Cristina Pineda de Carias y Bosque Sendra, J. (Edits.) Actas. I Congreso internacional de Ordenamiento territorial y tecnologías de la información geográfica. Servicio de publicaciones de la UAH, Alcalá de Henares, Madrid, 2011, 13 p. ISBN: 978-84-8138-920-3.

- Bosque Sendra, J., Díaz Castillo, C. y Díaz Muñoz, M.A. (2001). "De la justicia espacial a la justicia ambiental en la política de localización de instalaciones para la gestión de residuos en la comunidad de Madrid". Boletín de la Real Sociedad Geográfica, t. CXXXVII-CXXXVIII, 2001-2002, pp. 89-114. 
- Clifford, Geertz (1995). La interpretación de las culturas. Gedisa Barcelona Capítulo Descripción Densa, hacia una teoría interpretativa de la cultura. Pp. 101126.

- Couceiro-Vidal, A. (2008). Enseñanza de la bioética y planes de estudios basados en competencias. Viguera Editores SL 2008. EDUC MED 2008; 11 (2): 69-76.

- Escobar, J., Sarmiento, Y. y Gordillo, M.P. (2008). "La enseñanza de la bioética general como aporte en la construcción de un pensamiento bioético en los maestros". Revista Colombiana de Bioética, Vol. 3 Edición Especial - Noviembre de 2008.

- Gardner, Howard (2005). Las cinco mentes del futuro. Paidós, Barcelona, 2005.

- González, Ana Marta (2000). Ética y Moral. Origen de una diferencia conceptual y su trascendencia en el debate ético contemporáneo. Anuario Filosófico 2000, Servicio de Publicaciones de la Universidad de Navarra.

- González, J.L. y Martínez-Frías, J. (2011). “Geoética: un reto para la deontología profesional”. Tierra y Tecnología 39: 62-66.

- Herbert, Spencer (1992). "The principles of sociology". En. Bohannan Paul and Marx Glazar. Antropología Lecturas. Madrid. Mac Graw Hill.

- Keyeux, G., Penchaszadeh, V. y Saada, A. (2004). Ética de la Investigación en Seres Humanos y Políticas de Salud Pública. Organización de las Naciones Unidas para la Educación, la Ciencia y la Cultura (UNESCO) y Universidad Nacional de Colombia, ISBN: 958-701-745-5

- López López, Juan Carlos (2007). Teorías antropológicas. UNACH San Cristóbal de las Casas, Chiapas, México: UNACH PDF.

- Lovelock J. (2003) "The living Earth”. Nature 426, 769-770. 
- Martinez-Frias, J. (2008).“'Geoethics: proposal of a geosciences-oriented formal definition and future planetary perspectives". TIERRA: Spanish Thematic Network of Earth and Planetary Sciences. http://tierra.rediris.es. Redlris. TIERRA Documentos , 2008, 1.

- Martínez-Frías, J., González, J.L. \& Rull, F. (2012) "Geoethics and Deontology.From Fundamentals to applications in Planetary Protection". Episodes 34-4: 257-262.

- Martínez-Frías, J. y Pérez Verde, A. (2010) "Geoética, Meteoritos y Exploración Planetaria". La Tribuna. Astronomía128: 21.

- Mellado Rivera, Salvador (2004). Universidades y docencia en bioética. Edit. Club Universitario, ISBN: 978-84-8454-400-5.

- Méndez Pérez, F.J y Gómez Martínez, F.J. (2008). Educación Ético-Civica. Proyecto Ánfora, de Oxford EDUCACIÓN, 2008.

- Morin, Edgar (1999). Los siete saberes necesarios para la educación del futuo. Publicado en octubre de 1999 por la Organización de las Naciones Unidas para la Educación, la Ciencia y la Cultura - 7 place de Fontenoy - 75352 París 07 $\mathrm{SP}$ - Francia.

- Nemec, V. (1992). "Ethical Geology in the Education Process". 29th International Geological Congress, Kyoto, Japan, 24 August-3 September 1992. section II-24-1 «New ideas and techniques in geological education», v. 3, no. 3. Abstract/Paper 06.

- Nemec, V. (2012). "Geoethics and Sustainability". In: Proceedings of the 2nd World Sustain. Forum 1-30 November 2012; Sciforum Electronic Conferences Series, 2012. Nikitina, N. (2012). "Geoethics: theory, principles, problems". Monograph. M:, LLC Geoinformmark, 2012. 155 pp. ISBN 978-5-98877-049- 7?

- Potter, Van Rensselaer (1970). Bioethics: The science of survival. Perspectives in Biology and Medicine, Wisconsin University. 
- Riechmann, Jorge (2003). Tiempo para la Vida. La crisis ecológica en su dimensión temporal Ediciones del Genal, Málaga, ISBN: 84-932253-3-9.

- Riechmann, Jorge (2004). "Un apartheid planetario. Ecología, globalización y desigualdad socioeconómica". Conferencia inaugural del VI Congreso Vasco de Sociología pronunciada en Bilbao el 26 de febrero de 2004.

- Simondon, Gilbert (1989). L'individuation psychique et collective, París, Aubier, 1989.

- Swatz, M.J., Turnet V.W. and Tuden A. (1994). "Antropología Política. Una introducción". Alteridades sobre el conocimiento antropológico, № 8 , UAM-Iztapalapa, México. Pp. 101-126.

- Vasconcelos, L. (2012). "Geoethics". 1st Geological Congress of Mozambique, Maputo, Mozambique, 21-23 November, 2012, 1-11. 